\title{
Ein ultraschallbasiertes inverses Messverfahren zur Charakterisierung viskoelastischer Materialparameter von Polymeren
}

\author{
Fabian Bause, Bernd Henning \\ Elektrische Messtechnik, Universität Paderborn, Warburger Straße 100, \\ 33098 Paderborn, Deutschland
}

\section{Zusammenfassung}

Für die numerische Simulation von Schallausbreitungsphänomenen in akustischen (Mess-)Systemen ist die Verwendung realitätsnaher Materialmodelle und Materialparameter ein zentrales Problem. Akustische Materialparameter lassen sich nicht einfach aus quasistatisch ermittelten Kenngrößen, wie sie in Datenblättern angegeben werden, berechnen. Bestenfalls können grobe Abschätzungen getroffen werden, wobei diese gerade bei Polymeren für eine fundierte Betrachtung der Schallausbreitung unzureichend sind.

Es wird ein Messverfahren vorgestellt, welches, für eine gegebene polymere Materialprobe, ein komplex-wertiges und frequenzabhängiges Materialmodell, unter Berücksichtigung von Anisotropie sowie Spannungsrelaxations- und Retardationsprozessen, quantifiziert. Die Materialproben werden als hohlzylindrische Wellenleiter ausgelegt und Ultraschall-Transmissionsmessungen zwischen den parallelen Seiten der Probe durchgeführt [1]. Zur Berücksichtigung der Frequenzabhängigkeit werden fünf verschiedene Schallwandler-Paare mit einer aufsteigenden Mittenfrequenz von $750 \mathrm{kHz}$ bis $2,5 \mathrm{MHz}$ verwendet [2]. Jedes der fünf Messsignale trägt nach Durchlaufen der Materialprobe Informationen über die Materialparameter, die räumliche und spektrale Anregung der Probe sowie die Probengeometrie. In einem inversen Verfahren werden diese Informationen voneinander separiert. Die Lösung des inversen Problems erfolgt deterministisch durch iterativen Vergleich einer Vorwärtssimulation des gesamten Messsystems mit den experimentell bestimmten Messdaten.

Exemplarisch anhand des Werkstoffs Polyamamid 6 (PA6) wird das inverse Messverfahren vorgestellt sowie die Übertragbarkeit der damit gewonnenen Materialparameter auf konkurrierende Messverfahren diskutiert.

Keywords: Ultraschallmesstechnik, Polymere, Viskoelastizität, inverses Messverfahren

\section{Einleitung}

Die quantitative Beschreibung physikalischer Materialeigenschaften erfolgt über Materialmodelle. Im Gegensatz zu sogenannten Stoffgesetzen ist die Verwendung von Materialmodellen nicht zwingend eindeutig. So können für ein Material/Materialverhalten verschiedene Beschreibungsmodelle herangezogen werden, wobei jeweils deren Gültigkeitsgrenzen zu benennen sind. Einem Materialmodell sind zur quantitativen Abbildung von physikalischen Einflussgrößen auf dessen Reaktionsgrößen Materialparameter zugeordnet.

Für die numerische Simulation von Schallausbreitungsphänomenen in akustischen (Mess-)Systemen ist die Verwendung realitätsnaher Materialmodelle und Materialparameter ein zentrales Problem. Akustische Materialparameter lassen sich nicht einfach aus quasistatisch ermittelten
Kenngrößen, wie sie in Datenblättern angegeben werden, berechnen. Bestenfalls können grobe Abschätzungen getroffen werden, wobei diese gerade bei Polymeren für eine fundierte Betrachtung der Schallausbreitung unzureichend sind.

\section{Messung viskoelastischer Eigenschaften}

Es existieren viele verschiedene Prüfmethoden zur Charakterisierung der viskoelastischen Eigenschaften polymerer Werkstoffe. Eine Teilmenge dieser Verfahren wird im Folgenden skizziert. Grundlegend ist zu beachten, dass die Übertragbarkeit der Ergebnisse der verschiedenen Prüfmethoden nicht ohne weiteres gewährleistet ist. Zu hinterfragen ist z.B. stets der Einfluss des zur Prüfkörperherstellung verwendeten Verarbeitungsprozesses, welcher maßgeblich die morphologische Struktur des Prüfkörpers beeinflussen kann. Neben der 
chemischen Zusammensetzung, welche die unterschieden Polymere i.A. definieren, ist diese Struktur jedoch ebenfalls eine Einflussgröße. Es kann demnach nicht ein Polymer, welches auf Basis seiner chemischen Zusammensetzung charakterisiert ist, hinsichtlich seiner Eigenschaften geprüft werden, sondern es wird ein Prüfkörper auf Basis einer chemischen Zusammensetzung in einem durch den Verarbeitungsprozess (Spritzgießen, Extrudieren, Formpressen, Stanzen, Spanen, etc.) bedingten Zustand geprüft, siehe bspw. [3].

Zentrale Bedeutung im Bereich der quasistatischen Prüfung hat der Zeitstandversuch nach DIN EN ISO 899 (Kriech- oder Retardationsversuch). Bei dem Versuch wird eine konstante mechanische Spannung auf den Prüfkörper gegeben. Die Prüfkörpergeometrie ist nach DIN EN ISO 527 als Schultergeometrie vom Typ 1A bzw. 1B definiert. Zur Applikation einer konstanten Spannung kann z.B. eine definierte Masse genutzt werden. Gemessene Kriechkurven beginnen zumeist einige Sekunden verzögert zur Spannungsaufprägung, da diese technisch nicht als idealer Sprung realisiert werden kann. Unter der Bedingung linearer Prozesse kann durch FourierTransformation der frequenzabhängige Verlust- sowie Speichermodul aus den Zeitdaten errechnet werden. Mit dieser Methode ist allerdings nur der Frequenzbereich unterhalb von ca. $1 \mathrm{~Hz}$ zu charakterisieren, siehe [3, 4].

Mit Hilfe von Geräten zur Dynamisch Mechanischen Analyse (DMA) können viskoelastische Eigenschaften nach DIN EN ISO 6721 bestimmt werden. Bei der DMA wird ein i.d.R. streifenförmiger Probekörper mit einer harmonisch oszillierenden mechanischen Spannung beaufschlagt. Die dabei auftretende Dehnung am Körper wird über eine Wegmessung aufgezeichnet. Ausgewertet werden das Amplitudenverhältnis von aufgeprägter Spannung und gemessener Dehnung und deren Phasendifferenz. Darüber lassen sich dann für die beaufschlagte Frequenz der komplexe E-Modul bzw. der Verlust- und Speichermodul berechnen. Der Frequenzbereich kommerzieller Geräte liegt i.A. bei unter $100 \mathrm{~Hz}$. Eine Erweiterung des betrachteten Frequenzbereichs ist durch Ausnutzung der Zeit-Temperatur-Superposition prinzipiell möglich. Allerdings müssen dafür der Einfluss der Temperatur und der Zeit ungestört überlagert sein, was nur näherungsweise der Fall ist. Gerade deshalb sollte bei großen Extrapolationen seitens des Frequenzbereichs das Ergebnis kritisch hinterfragt werden $[3,4]$.

Die ultraschallbasierten Messmethoden zur Charakterisierung polymerer Eigenschaften lassen sich im Wesentlichen strukturieren in
DOI 10.5162/sensoren2016/5.3.1 Ebene-Wellen- und Geführte-Wellen-Ansätze. Diese sind wiederum in direkte und inverse Methoden zu gliedern, wobei nur für wenige einfache Versuchsaufbauten eine geschlossene Form zur direkten Berechnung gesuchter Materialparameter zugänglich ist.

Ein weit verbreiteter Ansatz zur Bestimmung der Materialparameter plattenförmiger, im Vergleich zur Wellenlänge großer, Probekörper mit ebenen Wellen ist die Immersionstechnik. Hier wird der zu untersuchende Körper in eine Drehvorrichtung eingespannt und in ein Wasserbad eingetaucht. Unter verschiedenen Winkeln wird nun die Transmission ebener Wellen durch den Körper untersucht [5,6,7]. Basierend auf einem Vorwärtsmodell (Christoffel Gleichung mit reellen Moduln [5,6] bzw. ThomsonHaskell-Matrix-Methode mit komplexen Moduln [7]) wird in einem inversen Verfahren ein Materialmodell des Probekörpers identifiziert.

Ein ähnlicher Ansatz wurde Anfang der 1990er Jahre von Sachse et al. unter dem Namen Point-Source/Point-Receiver Methode vorgestellt [8]. Ein Laser erzeugt räumlich stark begrenzt eine mechanische Welle welche mit einem Empfänger schmaler Apertur auf der gegenüberliegenden Seite des Prüfobjekts unter verschiedenen Winkeln bezogen auf den direkten Weg vom Sender zum Empfänger abgetastet wird. Durch die impulsförmige Anregung werden eine Quasi-Longitudinal- und zwei Quasi-Transversalwellen angeregt, deren Eintreffzeitpunkte am Empfänger separiert werden müssen. Mit Hilfe eines inversen Ansatzes kann das Materialmodell (hier reellwertige Moduln) identifiziert werden.

Bei Geführte-Wellen-Ansätzen wird gezielt die räumlich beschränkte Ausdehnung der Proben genutzt. Entsprechend ist die geometrische Form der Proben für das hinterlegte Modell von Bedeutung. Meist werden plattenförmige Probekörper genutzt, um entsprechend das dispersive Verhalten der symmetrischen und antisymmetrischen Lamb-Wellen auszuwerten. Im Wesentlichen unterscheiden sich GeführteWellen-Ansätze durch die gewählte Messmethode, d.h. den instrumentellen Einsatz, zur Bestimmung der dispersiven Kenngrößen des Wellenleiters. Zum Einsatz kommen Laserquellen [9] sowie Laser-Doppler-Vibrometer $[9,10]$, piezoelektrische Folienwandler, oder auch einfache piezokeramische Wandler [11]. Einen guten Überblick bietet hier die Arbeit von Chimenti [12].

Im Gegensatz zu plattenförmigen Wellenleiterstrukturen ist die Literaturlage hinsichtlich der Materialcharakterisierung auf Basis zylindrischer Probekörper deutlich dünner besetzt. Dies liegt nicht zuletzt an den zahlreichen Arbeiten bezüglich der Charakterisierung von 
gewalzten Metallplatten und plattenförmigen Kompositstrukturen. Extrudierte Stäbe (und auch Platten) auf Thermoplast-Basis spielen wirtschaftlich eine bedeutende Rolle, wenn aus technischen oder wirtschaftlichen Gründen kein Spritzgussverfahren eingesetzt werden kann. Die extrudierten Halbzeuge können nach dem Abkühlen spanend bearbeitet oder auch warm umgeformt werden. Gerade im Falle kleiner Stückzahlen, komplexer Geometrien oder großer Wandstärken wird dieses Verfahren häufig angewendet.

Einen ersten Ansatz zur Messung von Longitudinal- und Transversalwellengeschwindigkeit in einem zylindrischen Probekörper stellte Reynolds bereits im Jahr 1953 vor [13]. Der Durchmesser des Stabs wurde als groß gegenüber der betrachteten Wellenlänge beschrieben, sodass ein Strahlenansatz ohne Berücksichtigung von Wellenleitermoden angesetzt werden kann. Identifiziert wurde in einem direkten Verfahren ein isotropes elastisches Modell von metallischen Proben. Rautenberg griff diesen Ansatz 2012 wieder auf, um stark absorbierende Kunststoffe zu charakterisieren [1]. Die Probekörper wurden deutlich verkleinert, sodass wellentheoretische Ansätze zur Lösung des Wellenleiterproblems notwendig wurden, jedoch konnte der Ansatz von Reynolds zur Gewinnung von Startwerten für das inverse Problem, welches mit dem NelderMead-Simplex-Algorithmus gelöst wurde, verwendet werden. Identifiziert wurde von Rautenberg ein elastisches isotropes und ein transversal isotropes (mit quasi-isotroper Näherung) Materialmodell sowie zwei Dämpfungsparameter, welche zum RayleighDämpfungsmodell gehören.

Die Arbeiten von Rautenberg bilden die Grundlage für die im Folgenden beschriebene Arbeit.

\section{Materialmodell}

Im eindimensionalen Fall lässt sich viskoelastisches Verhalten im Frequenzbereich recht anschaulich beschreiben mit

(1) $\frac{\widetilde{\sigma}(i \omega)}{\tilde{\varepsilon}(i \omega)}=\tilde{G}(i \omega)=G^{\prime}(\omega)+i G^{\prime \prime}(\omega)$,

wobei $\tilde{G}(i \omega)$ der komplexe Modul und $G^{\prime}(\omega)$ der Speichermodul sowie $G^{\prime \prime}(\omega)$ der Verlustmodul beschreiben. Zur Beschreibung der Frequenzabhängigkeit sowie des Zusammenhangs von Speichermodul und Verlustmodul (Kausalität) wird, können verschiedene Materialmodelle angesetzt werden. Einen Überblick liefert z.B. [14]. Im Folgenden wird das fraktionale Zener-Modell verwendet, welches sowohl Spannungsrelaxation (Zeitkonstante $\tau_{\sigma}$ ) als auch Retardationsvorgänge (Zeitkonstante $\tau_{\varepsilon}$ ) im Material abbildet.

(2) $\quad \tilde{G}(i \omega)=E \frac{1+\left(i \omega \tau_{\varepsilon}\right)^{v}}{1+\left(i \omega \tau_{\sigma}\right)^{v}}$
DOI 10.5162/sensoren2016/5.3.1 Der Übergang von einer Dimension zur Dreidimensionalität wird durch die Zerlegung der quasi-isotropen Materialmatrix in seine orthogonalen Bestandteile realisiert (spektrale Zerlegung nach Theocaris). Es werden dann den Eigen-Kompressions- (Index K) und EigenScherbewegungen (Index G) unterschiedliche Verlustmechanismen zugeordnet. Es ergibt sich die komplexe Steifigkeitsmatrix zu:

$$
\begin{gathered}
\text { (3) } \widetilde{\boldsymbol{C}}(i \omega)=\left[\mu_{T} \boldsymbol{D}_{1}+\mu_{L} \boldsymbol{D}_{2}+\frac{E_{L}}{1+v_{L}} \boldsymbol{D}_{3}\right] \\
\frac{1+\left(i \omega \tau_{\varepsilon, G}\right)^{v, G}}{1+\left(i \omega \tau_{\sigma, G}\right)^{v, G}}+\frac{E_{L}}{1+2 v_{L}} \boldsymbol{D}_{4} \frac{1+\left(i \omega \tau_{\varepsilon, K}\right)^{v, K}}{1+\left(i \omega \tau_{\sigma, K}\right)^{v, K}}
\end{gathered}
$$

Details zur Zerlegung und Strukturierung des Materialmodells finden sich in [2]. Der erste Teil von Gl. (3) mit den Projektionsmatrizen $\boldsymbol{D}_{1}$ bis $D_{3}$ beschreibt Scherbewegungen, hingegen beschreibt der zweite Part mit der Projektionsmatrix $\boldsymbol{D}_{4}$ reine Dilatationsbewegungen.

\section{Beschreibung des Versuchsaufbaus}

Der Versuchsaufbau besteht aus einer einfachen Transmissionstrecke. Piezoelektrische 13-Kompositwandler werden auf den parallelen Seiten einer zylindrischen Polymerprobe aufgesetzt und der Probenkörper durchschallt, vgl. Abb. 1 (links). Um die Frequenzabhängigkeit des Polymers bewerten zu können, werden insgesamt fünf Schallwandler-Paare mit einer aufsteigenden Mittenfrequenz von 750 $\mathrm{kHz}$ bis $2,5 \mathrm{MHz}$ genutzt $(750 \mathrm{kHz} ; 1 \mathrm{MHz}$; $1,5 \mathrm{MHz}, 2 \mathrm{MHz} ; 2,5 \mathrm{MHz}$ ). Die relative Bandbreite der Wandler beträgt ca. $60 \%$.

Der Aufbau (Sender, Probe und Empfänger) wird in einer klimatisierten Kammer betrieben, sodass sichergestellt werden kann, dass alle Messungen unter identischer Temperatur getätigt werden.

In Abb. 1 (rechts) ist ein typisches Empfangssignal zu erkennen. Durch die Dispersion im Probekörper ist der Sendepuls zeitlich zerlaufen. Durch die spezielle Wahl der Geometrie entstehen mehrere zeitlich separierbare Pulse im Empfangssignal, was der Sensitivität auf die Messgrößen sowie der Startwertbestimmung für das inverse Verfahren dienlich ist.
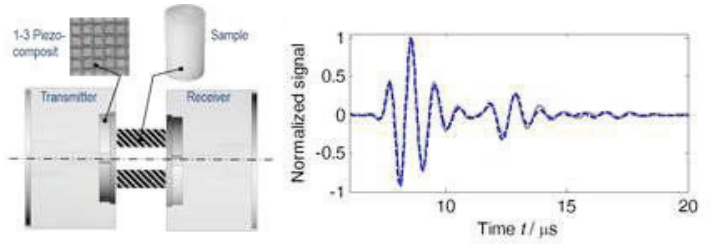

Abb. 1: Transmissionsstrecke (links) und beispielhaftes Empfangssignal (rechts). 


\section{Inverses Messverfahren}

Das Messverfahren steht auf drei Säulen:

- Vorwärtsmodell zur Simulation der Empfangssignale unter Annahme bestimmter Materialparameter

- Optimierungsalgorithmus zur iterativen Adaption der simulierten Materialparameter bis zur bestmöglichen Übereinstimmung von simulierten und gemessenen Werten

- Unsicherheitsanalyse der Ergebnisse sowie der Diskussion deren Übertragbarkeit

Eine ausführliche Betrachtung der einzelnen Stationen des Messverfahrens ist in [2] beschrieben.

Das Vorwärtsmodell besteht zunächst aus den Modellen der

- Schallwandler (Sender sowie Empfänger)

- Sende- und Empfangsverstärker

- Koaxialleitungen

Die Modelle können separat identifiziert werden, wobei seitens der Sendeverstärkung stets die Rückkopplung zwischen elektrischer Last des jeweilig angeschlossenen Sendewandlers auf die Übertragungseigenschaften des Verstärkers beachtet werden muss. Die Schallwandler wurden basierend auf deren elektrischer Impedanz und dem Mason-Modell identifiziert. Die Koaxialleitungen wurden als Leitungsgleichungen modelliert und auch über die elektrische Impedanz parametrisiert. Die Identifikation der Übertagungseigenschaften der Verstärker wurde mit dem Steiglitz-McBrightAlgorithmus realisiert [15].

Das akustische Vorwärtsmodell, d.h. die Simulation der Schallausbreitung in der Probe, wird realisiert durch Berechnung der komplexen Dispersionsdiagramme mit der ScaledBoundary- FEM [16], gekoppelt mit der Methode der modalen Expansion [2, 17,18] zur Berechnung transienter Empfangssignale.

Die Optimierung der Materialparameter wurde mit dem BOBYQA-Algorithmus von Powell durchgeführt [19]. Der Name BOBYQA ist ein Akronym für Bounded Optimization BY Quadratic Approximation und bietet sich an bei Optimierungsproblemen bei denen keine Ableitungsinformationen zur Verfügung stehen sowie die einzelnen Funktionsauswertungen (Vorwärts-Simulationen) rechenkosten-intensiv sind.

Die Unsicherheitsanalyse der Ergebnisse wurde realisiert basierend auf den Vorgaben des Guide to the Expression of Uncertainty in Measurement (GUM) des Joint Committee for Guides in Metrology (JCGM). Das Vorwärtsmodell wurde dazu linearisiert und so umgestellt, dass das GUM-Verfahren angewendet werden kann $[20,2]$.
DOI 10.5162/sensoren2016/5.3.1

Zur Überprüfung der Übertragbarkeit der Messergebnisse wurde ein weiterer Messplatz aufgebaut, welcher die Untersuchung der Lamb-Wellen-Ausbreitung in plattenförmigen Strukturen ermöglicht [21]. Eine Skizze des Versuchsaufbaus ist in Abb. 2 zu sehen. Die ausbreitungsfähigen Lamb-Wellen können mit dem Messplatz in Dispersionsabbildungen charakterisiert werden.

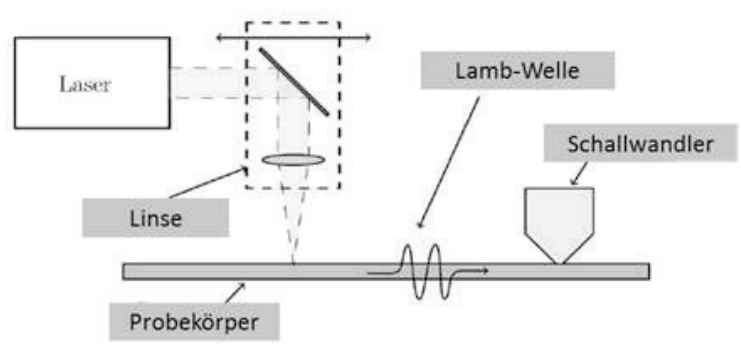

Abb. 2: Aufbau des Laser-AkustikMessplatzes, Abb. nach [18].

\section{Ergebnisse}

Im Folgenden werden Ergebnisse für die Untersuchung von Polyamid 6 (PA6) dargelegt. In den Abbildungen 3 und 4 ist das frequenzabhängige Verhalten der Longitudinal- und Transversalwelle aufgezeigt. Bezogen auf die Materialmatrix beziehen sich diese Wellenformen auf die 33- bzw. 66-Komponente der Matrix. Dargestellt sind Schätzwerte für die Phasengeschwindigkeit sowie den Absorptionskoeffizienten bei den Mittenfrequenzen der Schallwandler (rote Kreise). Diese Schätzwerte werden direkt aus den transienten Empfangssignalen gewonnen. Basierend auf diesen Schätzwerten wird ein initiales Materialmodell parametrisiert, welches durch die rote durchgezogene Linie dargestellt wird. Das endgültige Ergebnis nach der Optimierung, d.h. das Ergebnis des inversen Verfahrens, ist in grün dargestellt.

Die Messunsicherheit wird dargestellt als symmetrische 95\%-Konfidenzgrenzen. Als Ursachen der Messunsicherheit werden berücksichtigt: Messrauschen, Trigger-Jitter, Geometrie-Unsicherheiten der Proben sowie Unsicherheiten der Justierung des Messsystems (im Wesentlichen Unsicherheit der Koppelschichtdicke).

Dargestellt werden sowohl die absolute Unsicherheit (blau) sowie die relative Unsicherheit (grün), wobei der Bezug auf den jeweiligen Schätzwert bei der betrachteten Frequenz zu verstehen ist, vgl. Abb. 5 und Abb. 6. 

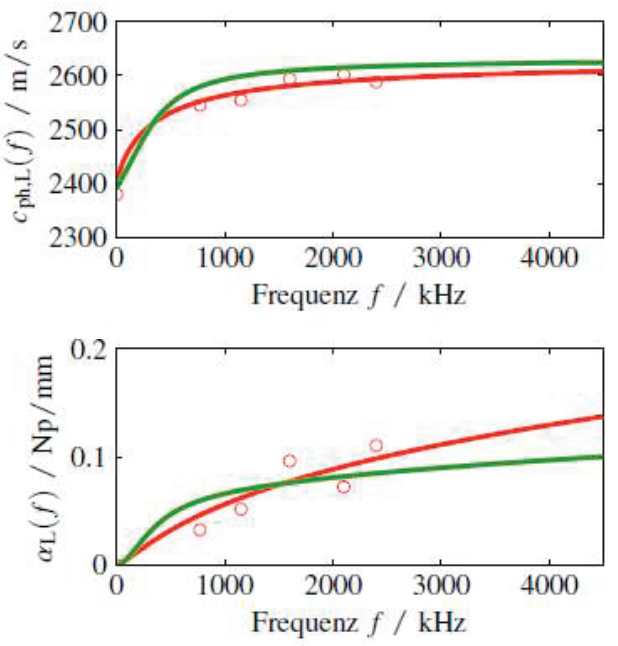

Abb. 3: Ergebnis der Untersuchung für PA6 (Longitudinalwellengeschwindigkeit). Rote Kreise: Diskrete Schätzungen aus den fünf Empfangssignalen. Rote Linie: Modellbasierte Schätzung auf Basis der roten Kreise. Grün: Ergebnis nach der Optimierung.
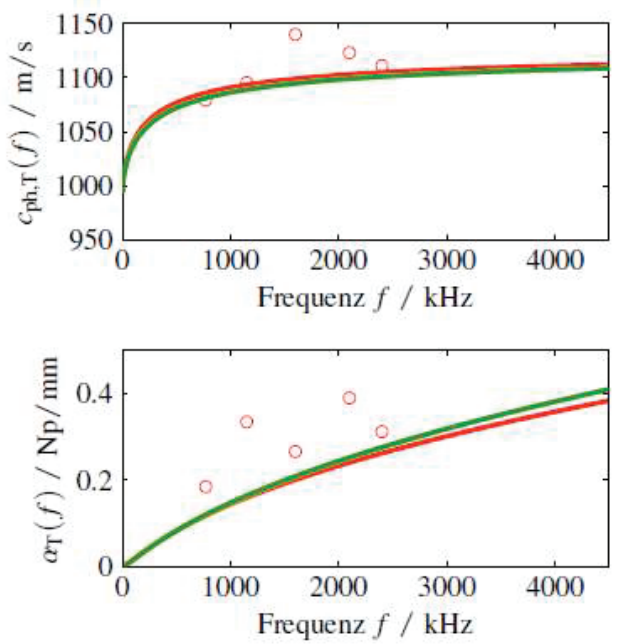

Abb. 4: Ergebnis der Untersuchung für PA6 (Transversalwellengeschwindigkeit). Rote Kreise: Diskrete Schätzungen aus den fünf Empfangssignalen. Rote Linie: Modellbasierte Schätzung auf Basis der roten Kreise. Grün: Ergebnis nach der Optimierung.

Die Materialparameter definieren sich durch inre Unabhängigkeit von der Geometrie des Körpers. Entsprechend ist eine Überprüfung der Materialparameter mit geänderter Probengeometrie sowie mit anderen Messmethoden ein probates Mittel zur Überprüfung des vorgestellten Messverfahrens. Abb. 7 zeigt in Farbstufen die Dispersionsabbildung einer PA6Platte. In roten Kreisen wurde eine Simulation der Dispersionseigenschaften der Platte mit
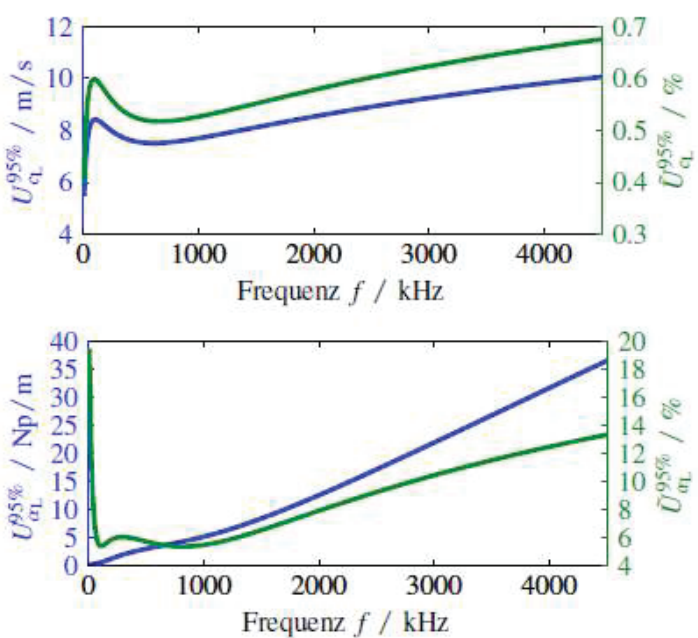

Abb. 5: Unsicherheitsanalyse der erzielten Ergebnisse für die Longitudinalwelle. Blau: Absolut, Grün: Relativ bezogen auf den Schätzwert.
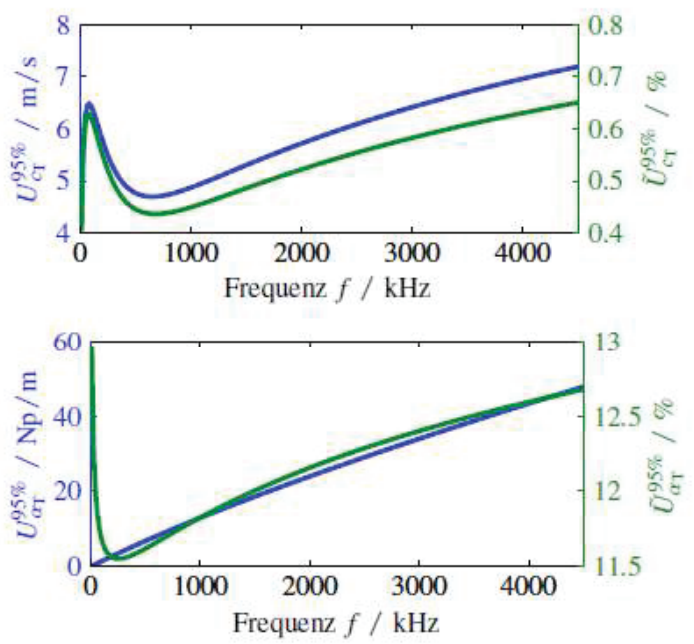

Abb. 6: Unsicherheitsanalyse der erzielten Ergebnisse für die Pure Transversalwelle (66-Komponente der Materialmatrix). Blau: Absolut, Grün: Relativ bezogen auf den Schätzwert.

den zuvor bestimmten Materialparametern überlagert. Es zeigt sich eine gute Übereinstimmung zwischen der Messung und der Simulation. Durch die starke Dämpfung des Materials und der langen Laufwege der Schallwellen in der Platte ist diese Methode auf den unteren Frequenzbereich beschränkt. 


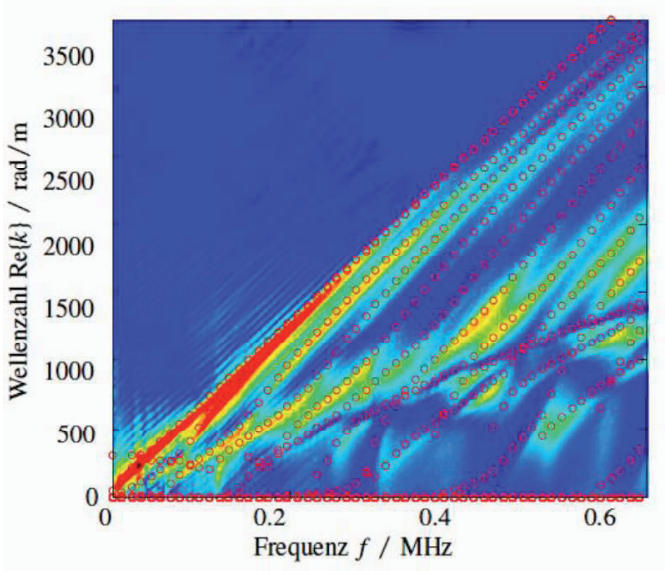

Abb. 7: Vergleich zwischen Laserakustischer Messung und den ermittelten Materialparametern.

Zur Validierung mit einem standardisierten Messverfahren wurde eine DMA im 3-PunktBiege-Modus durchgeführt. Problematisch ist, dass diese Bewegungsform keine reine Scherung darstellt und deshalb der Vergleich schwierig ist. Durch lineare Umrechnungen wurde aus dem Speicher- und Verlustmodul der DMA die Absorption der Transversalwelle berechnet, siehe Abb. 8 (rot). Die blaue durchgezogene Linie repräsentiert den besten Schätzwert sowie die 95\%-Konfidenzgrenzen (unterbrochene Linien) aus dem vorgestellten Messverfahren.

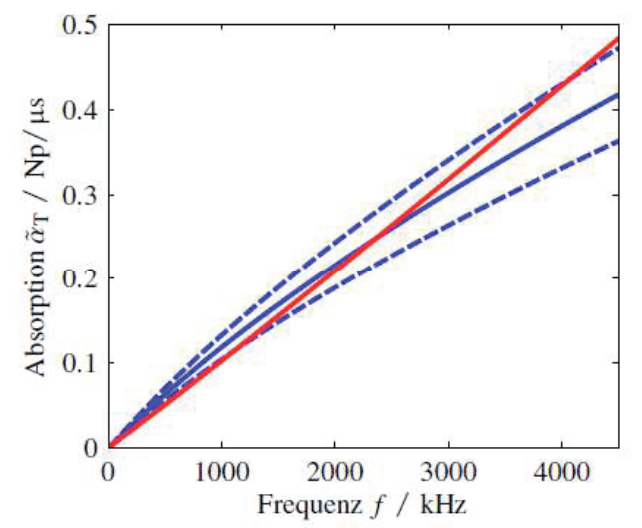

Abb. 8: Vergleich zwischen DMA (rot) und den ermittelten Eigenschaften (blau) aus dem vorgestellten Messverfahren.

\section{Danksagung}

Die Autoren bedanken sich bei der DFG für die Förderung des Forschungsprojekts HE 2897/31 (Bestimmung komplexer akustischer Materialdaten).

\section{Literaturnachweis}

[1] Rautenberg, J.: Ein wellenleiterbasiertes Verfahren zur Bestimmung von Materialdaten für die realitätsnahe Simulation von Schallausbreitungsphänomenen am Beispiel stark absorbierender Kunststoffe. Dissertation. Universität Paderborn (2012)

[2] Bause, F.: Ein ultraschallbasiertes inverses Messverfahren zur Charakterisierung viskoelastischer Materialparameter von Polymeren. Dissertation. Universität Paderborn. (Laufendes Verfahren)

[3] W. Grellmann und S. Siedler: Kunststoffprüfung, 2. Auflage. München: Hanser (2011)

[4] R. Lakes: Viscoelastic measurement techniques. Review of Scientific Instruments, 75.4 (2004), S. 797-810

[5] S. S. S. Reddy, K. Balasubramaniam, C. Krishnamurthy und M. Shankar: Ultrasonic goniometry immersion techniques for the measurement of elastic moduli. Composite Structures, 67.1 (2005), S. 3-17

[6] J. Vishnuvardhan, C. Krishnamurthy und K. Balasubramaniam: Genetic algorithm reconstruction of orthotropic composite plate elastic constants from a single nonsymmetric plane ultrasonic velocity data. Composites Part B: Engineering, 38.2 (2007), S. 216-227

[7] M. Castaings, B. Hosten und T. Kundu: Inversion of ultrasonic, plane-wave transmission data in composite plates to infer viscoelastic aterial properties. NDT \& E International, 33.6 (2000), S. 377-392

[8] B. Castagnede, K. Y. Kim, W. Sachse und M. Thompson: Determination of the elastic constants of anisotropic materials using laser generated ultrasonic signals. Journal of Applied Physics, 70.1 (1991), S. 150-157

[9] F. Singer: Mess- und Analysemethoden in der Laserakustik bei breitbandiger Laseranregung. tm - Technisches Messen, 82.1 (2015), S. 4551

[10] K. Heller, L. Jacobs und J. Qu: Characterization of adhesive bond properties using Lamb waves. NDT \& E International, 33.8 (2000), S. 555-563

[11] A. Marzani und L. De Marchi: Characterization of the elastic moduli in composite plates via dispersive guided waves data and genetic algorithms. Journal of Intelligent Material Systems and Structures, 24.17 (2012), S. 21352147

[12] D. E. Chimenti: Guided waves in plates and their use in materials characterization. Applied Mechanics Reviews, 50.5 (1997), S. 247-284

[13] M. Reynolds: The Determination of the Elastic Constants of Metals by the Ultrasonic Pulse Technique. Transactions American Society for Metals, 45 (1953), S. 839-861

[14] F. Mainardi: Fractional Calculus and Waves in Linear Viscoelasticity - An Introduction to Mathematical Models. London: Imperial College Press (2010)

[15] K. Steiglitz und L. McBride: A Technique for the Identification of Linear Systems. EEE Transactions on Automatic Control, 4 (1965), S. 461646 
[16] H. Gravenkamp, Numerical methods for the simulation of ultrasonic guided waves, Dissertation, BAM-Dissertationsreihe, Band 116 (2014)

[17] A. Puckett, M. Peterson, A semi-analytical model for predicting multiple propagating axially symmetric modes in cylindrical waveguides, UItrasonics 43.3 (2005) S. 197-207

[18] F. Bause, H. Gravenkamp, J. Rautenberg, B. Henning: Transient modeling of ultrasonic guided waves in circular viscoelastic waveguides for inverse material characterization. Measurement Science and Technology, 26 (2015)

[19] M. Powell: The BOBYQA algorithm for bound constrained optimization without derivatives. Department of Applied Mathematics und Theoretical Physics, Cambridge, technical report DAMTP 2009/NA06 (2009)

[20] JCGM - Joint Committee for Guides in Metrology: JCGM 102:2011 - Evaluation of measurement data - Supplement 2 to the "Guide to the expression of uncertainty in measurement"- Extension to any number of output quantities. (2011)

[21] L. Claes, F. Bause, J. Rautenberg und B. Henning: Detection of ultrasonic plate waves using ceramic strip transducers. Sensor Conferences Nuremberg. (2015), Proceedings, S. 775-779 